\title{
Usabilidade de aplicativo móvel em saúde: uma revisão bibliométrica
}

\author{
Usability of a mobile health application: a bibliometric review
}

Usabilidad de una aplicación de salud móvil: una revisión bibliométrica

\begin{abstract}
Louyse Victória Ferreira da Silva ${ }^{1 *}$, Joelma Silva Santos ${ }^{1}$, Ana Letícia Alves de Carvalho ${ }^{1}$, Daniele Mamédio de Andrade ${ }^{1}$, Davi Dantas de Sá1, Érica Pereira Alves ${ }^{1}$, Fabiola Kelli Silva Felix¹, Letícia Lany de Miranda Medeiros ${ }^{1}$, Beatriz Mamedio de Andrade ${ }^{2}$, Taciana da Costa Farias Almeida ${ }^{1}$.
\end{abstract}

\section{RESUMO}

Objetivo: Caracterizar a produção científica relacionada à avaliação da usabilidade de aplicativos na área da saúde. Métodos: Trata-se de um estudo bibliométrico, quantitativo e descritivo. As variáveis de interesse para este estudo foram: base de dados, ano de publicação, periódico, autores, titulação de autores, Qualis/Capes, fator de impacto, instrumento utilizado para medir a usabilidade e área de aplicação. Resultados: Foram considerados elegíveis para a análise 18 artigos. Identificou-se que 77,8\% estavam disponíveis na MEDLINE; 55,5\% publicados no ano de 2019; $77,8 \%$ no idioma Inglês; $82,3 \%$ publicações internacionais; $66,7 \%$ apresentando Qualis/Capes estrato "A"; $66,7 \%$ de autoria multiprofissional, com uma diversidade de aplicabilidade nas áreas da saúde, destacando-se as doenças crônicas; $72,2 \%$ utilizaram a System Usability Scale; e 94,4\% dos estudos foram considerados validados em relação à aplicabilidade de escalas de avaliação e usabilidade pelos usuários. Considerações finais: A caracterização dos estudos possibilitou um maior conhecimento acerca da temática usabilidade de App moveis em saúde, assim como evidenciou a inovação desse tipo de estudo, predominância do uso da System Usability Scale, importância de construções de tecnologias de forma interprofissional, uma vez que possibilita ao usuário um autocuidado de forma integral ao acessar aplicativos na área da saúde.

Palavras-chave: Usabilidade, Aplicativos móveis, Saúde.

\begin{abstract}
Objective: To characterize the scientific production related to the evaluation of the usability of applications in the health area. Methods: This is a bibliometric, quantitative and descriptive study. The variables of interest for this study were: database, year of publication, journal, authors, authors' titles, Qualis / Capes, impact factor, instrument used to measure usability and area of application. Results: 18 articles were considered eligible for analysis. It was identified that $77.8 \%$ were available on MEDLINE; $55.5 \%$ published in $2019 ; 77.8 \%$ in the English language; $82.3 \%$ international publications; $66.7 \%$ presenting Qualis / Capes stratum "A"; $66.7 \%$ of multidisciplinary authorship, with a diversity of applicability in the areas of health, with emphasis on chronic diseases; $72.2 \%$ used the System Usability Scale; and $94.4 \%$ of the studies were considered validated in relation to the applicability of evaluation scales and usability by users. Final considerations: The characterization of the studies enabled a greater knowledge about the usability theme of mobile App in health, as well as evidenced the innovation of this type of study, predominance of the use of the System Usability Scale, the importance of building technologies in an interprofessional way, since enables the user to provide full self-care when accessing healthcare applications.
\end{abstract}

Keywords: Usability, Mobile apps, Health.

\section{RESUMEN}

Objetivo: Caracterizar la producción científica relacionada con la evaluación de la usabilidad de aplicaciones en el área de la salud. Métodos: Se trata de un estudio bibliométrico, cuantitativo y descriptivo. Las variables de interés para este estudio fueron: base de datos, año de publicación, revista, autores, títulos de los autores, Qualis / Capes, factor de impacto, instrumento utilizado para medir usabilidad y área de aplicación. Resultados: Se consideraron elegibles para el análisis 18 artículos. Se identificó que el 77,8\% estaban disponibles en MEDLINE; 55,5\% publicado en 2019; 77,8\% en idioma inglés; $82,3 \%$ de publicaciones internacionales; $66,7 \%$ presenta Qualis / Capes estrato "A"; $66,7 \%$ de autoría multiprofesional, con diversidad de aplicabilidad en las áreas de salud, con énfasis en enfermedades crónicas; $72,2 \%$ utilizó la Escala de usabilidad del sistema; y el 94,4\% de los estudios se consideraron validados en relación con la aplicabilidad de las escalas de evaluación y la usabilidad por parte de los usuarios. Consideraciones finales: La caracterización de los estudios permitió un mayor conocimiento sobre el tema de usabilidad de la aplicación móvil en salud, así como evidenció la innovación de este tipo de estudios, predominio del uso de la Escala de usabilidad del sistema, la importancia de las tecnologías de construcción en un de forma interprofesional, permite al usuario cuidarse completamente a sí mismo al acceder a las aplicaciones sanitarias.

Palabras clave: Usabilidad, Aplicaciones móviles, Salud.

${ }^{1}$ Universidade Federal de Campina Grande (UFCG), Campina Grande - PB. *E-mail: victorialouyse@outlook.com

${ }^{2}$ Centro Universitário Unifacisa (UNIFACISA), Campina Grande - PB. 


\section{INTRODUÇÃO}

A disseminação da Internet sem fio e o uso de dispositivos móveis têm facilitado o acesso a informações em qualquer lugar e a todo momento, de modo a simplificar tarefas e oferecer subsídios para tomadas de decisões, através de aplicativos móveis. Este campo de possibilidades acompanha as inovações tecnológicas, e surgem os diferentes aplicativos móveis voltados para a área da saúde, com perspectiva de proporcionar autonomia nos cuidados, especialmente aqueles relacionados à sua continuidade nos ambientes extra hospitalar e ambulatorial (GAMA LN e TAVARES CMM, 2019).

O emprego de aplicativos (Apps) móveis voltados para a saúde pode atuar como ferramenta para o autogerenciamento de doenças e envolvimento do cliente no processo de autocuidado, seja na adesão a medicamentos, terapêuticas não farmacológicas ou realização de tarefas, o que facilita a obtenção de resultados clínicos na condução de tratamentos, proposta promissora em relação a motivar pacientes para o autocuidado no domicílio (JOHNSTON N, et al., 2016; PECORELLI N, et al., 2018).

Evidências científicas apontam a importância dessa ferramenta tecnológica como facilitadora no cuidado domiciliar, como constatado em estudo que identificou a importância de um aplicativo móvel utilizado por um grupo de pacientes submetidos a cirurgia intestinal. O App foi utilizado como ferramenta de apoio à recuperação cirúrgica e como registro de adesão do paciente a um programa de recuperação aprimorada, o qual identificou que $89 \%$ dos participantes relataram que o aplicativo foi útil para atingir objetivos de recuperação, e $76 \%$ ainda acrescentaram que o aplicativo foi um incentivo para a recuperação pós-cirúrgica (PECORELLI N, et al., 2018).

Pacientes, instituições e profissionais da saúde já utilizam aplicativos móveis, como forma de interligar contextos, usuários e saberes, além de ampliar estratégias, em que cuidar e educar avançam juntos (GAMA LN e TAVARES CMM, 2019). Entretanto, o desenvolvimento de Apps móveis e sua distribuição em plataformas digitais, com diferentes finalidades e variadas funcionalidades, não são suficientes para garantir eficácia, eficiência e validade na utilização dos recursos oferecidos pela tecnologia. Assim, ao propor um App que tenha como proposta melhorar os cuidados em saúde, são imprescindíveis os desdobramentos de etapas metodológicas compactadas que promovam a construção desses App e que atestem as funcionalidades da tecnologia empregada e, dentro deste processo, encontra-se a avaliação da usabilidade (ALVIM AL e COUTO B, 2019).

A usabilidade de Apps móveis compreende a forma de avaliar a facilidade de utilização, a maneira como os indivíduos interagem com o sistema, medida por intermédio de instrumentos validados, e a capacidade de um programa/aplicativo ser compreendido, entendido e operacionalizado por um indivíduo, atingindo sua finalidade específica (SANTOS AO, et al., 2019; LIMA CJM, et al., 2019).

Nesse sentido, pergunta-se: Quais as características dos estudos de usabilidade de App móveis, na área da saúde, em relação as escalas de usabilidade aplicadas? Assim, estudos que busquem avaliar a usabilidade de Apps móveis em saúde são relevantes e precisam ser publicados para o conhecimento da população sobre eficácia, eficiência e validade do que se propõe, para que sejam utilizados com segurança, afirmando a aceitação pelo público-alvo do App. Diante do exposto, buscou-se, com este estudo, caracterizar a produção científica relacionada à avaliação da usabilidade de aplicativos na área da saúde.

\section{MÉTODOS}

Trata-se de um estudo bibliométrico com abordagem quantitativa. A bibliometria é composta por leis e princípios empíricos estatísticos que colaboram para determinar a fundamentação teórica da área da ciência da informação. A aplicação dessas leis e princípios possibilita a produção de diferentes indicadores relevantes para o tratamento e a gestão da informação e do conhecimento (GUEDES VLS, 2012; MEDEIROS KKAS, et al., 2012).

Para a realização deste estudo, algumas etapas foram seguidas, a saber: 1. Levantamento dos dados por meio de buscas nas bases de dados; 2 . Identificação das variáveis de interesse para o estudo; 3 . Leitura de títulos, resumos e texto completo por duas pesquisadoras; 4 . Organização dos indicadores relevantes; 5 . Tratamento dos dados para responder ao objetivo proposto. 
Elencaram-se as bases de dados: Sistema Online de Busca e Análise de Literatura Médica (MEDLINE) e Literatura Latino-Americana e do Caribe em Ciências da Saúde (LILACS), para o levantamento dos dados, durante os meses de setembro e outubro de 2020. Foram utilizados os descritores "Usability" e "App" na MEDLINE, e "Usabilidade" e "App" na LILACS. Em ambas as buscas, com a interposição do operador AND. Inicialmente, procurou-se estabelecer critérios de leitura do título para a investigação, objetivando remover artigos que não se encaixavam na temática; e, com a finalidade de buscar por literatura atual, utilizou-se o recorte de tempo de publicação de cinco anos (2016-2020).

Como critérios de inclusão, adotaram-se textos no formato de artigos que abordassem a verificação da usabilidade de aplicativos móveis na área da saúde, publicados nos idiomas português, inglês e espanhol, do tipo ensaio clínico, meta-análise ou teste controlado e aleatório. Foram excluídos textos não científicos, capítulos de livros, artigos de revisão, monografias, dissertações ou teses e estudos de revisão.

Foram elencadas como variáveis de interesse para este estudo: título, base de dados, ano de publicação, periódico, autores, titulação de autores, Qualis/Capes, Fator de Impacto, local de realização do estudo, tipo de estudo, instrumento utilizado para medir a usabilidade e área de aplicação; as quais foram inseridas em planilha criada no programa Microsoft Excel®, e preenchidas após a leitura crítica dos artigos por duas pesquisadoras.

Após a leitura dos títulos, resumos e texto completo por duas pesquisadoras, foram selecionados os artigos que compuseram a amostra, e excluídos aqueles que não testaram a usabilidade (Figura 1).

Figura 1 - Diagrama do processo de seleção das produções científicas sobre a avaliação da usabilidade de aplicativos móveis.

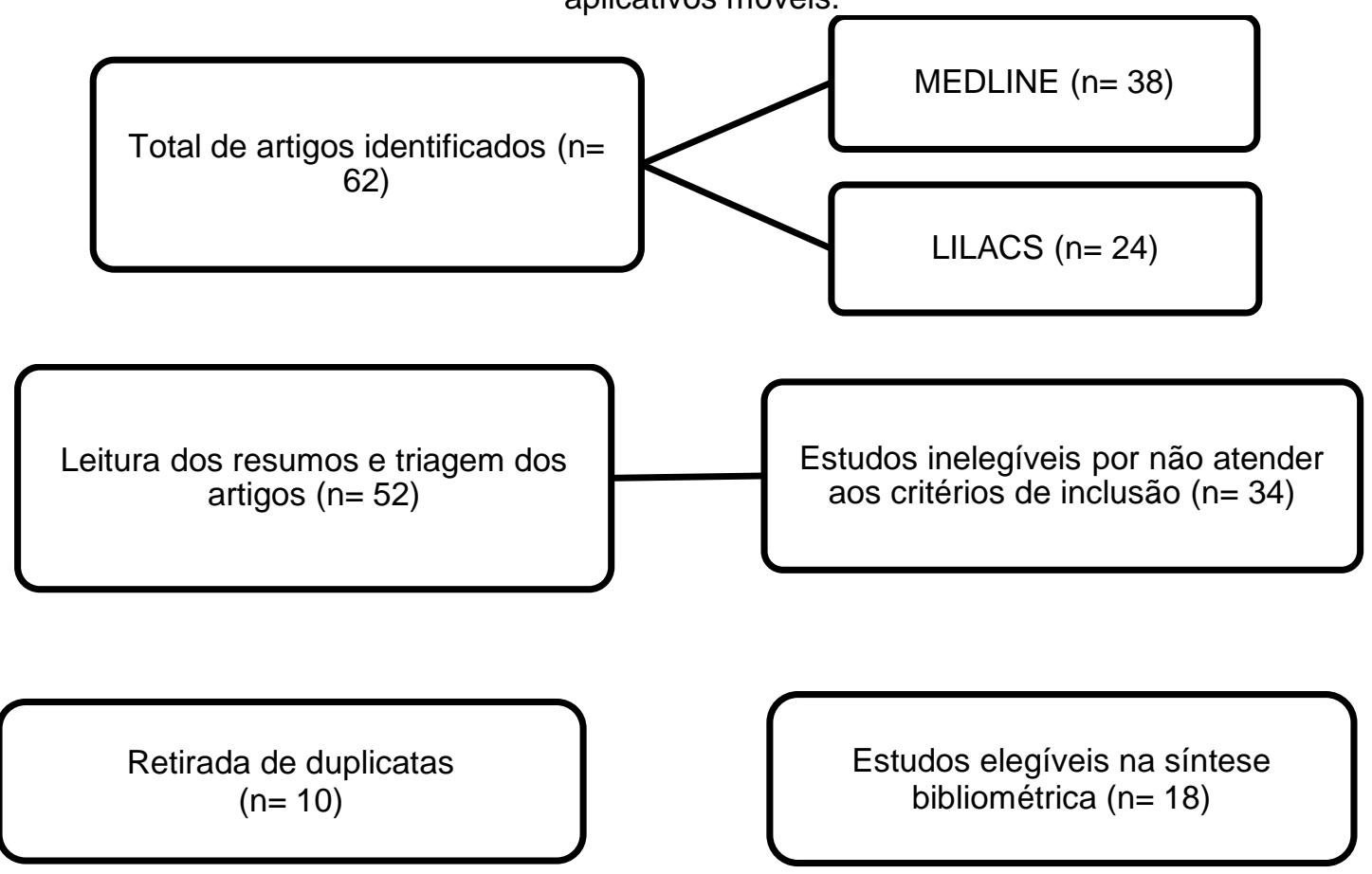

Fonte: Silva LVF, et al., 2021.

\section{RESULTADOS}

Ao examinar os dados das variáveis bibliométricas dos 18 artigos selecionados, observou-se que 14 $(77,8 \%)$ estudos estavam disponíveis na MEDLINE e quatro (22,2\%), na LILACS. Para os idiomas, 14 $(77,8 \%)$ foram publicados em inglês e quatro $(22,2 \%)$, em português.

Com relação à concentração de publicações de artigos por ano, predominou o ano de 2019 com 10 (55,5\%), seguido do ano de $2018 \mathrm{com}$ quatro (22,2\%). Não foram identificadas publicações, de acordo com os critérios estabelecidos para este estudo, no ano de 2017 (Figura 2). 
Figura 2 - Distribuição dos estudos conforme o uso de escalas para usabilidade, quanto ao ano de publicação, no período de 2016 a 2020.

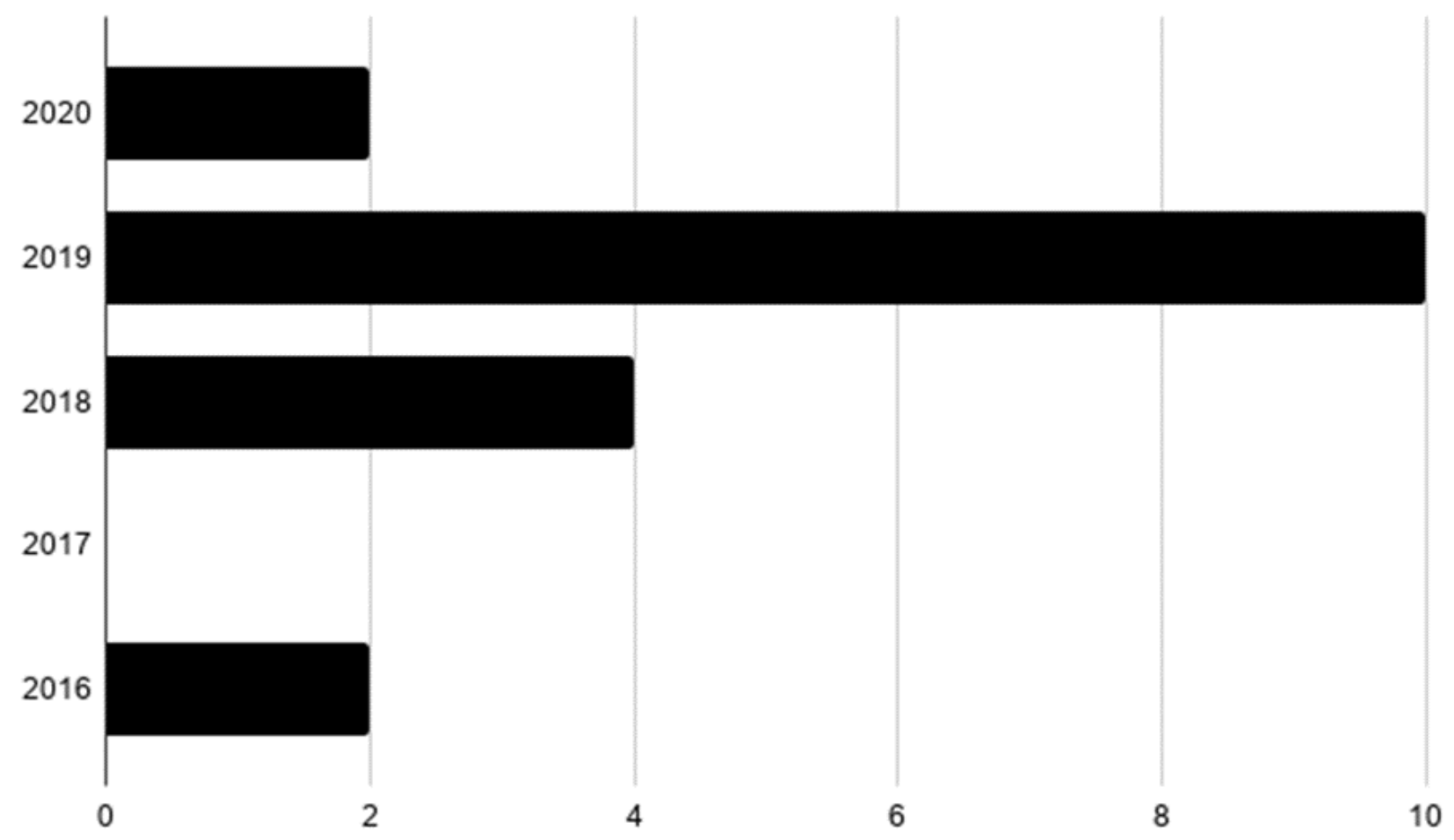

Fonte: Silva LVF, et al., 2021.

Quanto à disposição dos periódicos científicos, 14 (82,3\%) são internacionais e três, nacionais (17,6\%), com destaque para a Revista Brasileira de Educação Médica, com duas publicações. Todos os estudos apresentavam Qualis/Capes/Brasil - Plataforma Sucupira (2013-2016), e sua organização compreendia variedade. Quanto ao fator de impacto, não foi identificado para três periódicos, sendo o maior valor atribuído ao jornal acadêmico Thorax (Tabela 1).

Tabela 1 - Distribuição dos periódicos com publicações de estudos com os critérios de avaliação estabelecidos (QUALIS CAPES 2013-2016/ FATOR DE IMPACTO-JCR).

\begin{tabular}{lccc}
\hline Periódico & $\mathbf{n}$ & Qualis Capes & Fator de impacto \\
\hline American Heart Journal & 01 & $\mathrm{~A} 1$ & 4.153 \\
Behavior Therapy & 01 & $\mathrm{~A} 1$ & 3.243 \\
The International Journal of Behavioral Nutrition and & 01 & $\mathrm{~A} 1$ & 6.714 \\
Physical Activity & 01 & $\mathrm{~A} 1$ & 5.034 \\
JMIR Journal of Medical Internet Research & 01 & $\mathrm{~A} 1$ & 8.834 \\
Thorax & 01 & $\mathrm{~A} 2$ & 3.025 \\
International Journal of Medical Informatics & 01 & $\mathrm{~A} 2$ & 2.139 \\
International Journal of Behavioral Medicine & 01 & $\mathrm{~A} 2$ & 2.503 \\
Journal of Health Psychology & 01 & $\mathrm{~A} 2$ & 2.513 \\
Pain Medicine & 01 & $\mathrm{~A} 2$ & 2.635 \\
Supportive Care in Cancer & 01 & $\mathrm{~A} 2$ & 3.149 \\
Surgical Endoscopy & 01 & $\mathrm{~A} 2$ & $*$ \\
Texto \& Contexto Enfermagem & 01 & $\mathrm{~B} 2$ & 3.088 \\
Addiction Science \& Clinical Practice & 02 & $\mathrm{~B} 2$ & $*$ \\
Revista Brasileira de Educação Médica & 01 & $\mathrm{~B} 2$ & $*$ \\
Revista Enfermagem em Foco & 01 & $\mathrm{~B} 2$ & 2.864 \\
Translational Behavioral Medicine & 01 & $\mathrm{~B} 5$ & 4.313 \\
JMIR Mhealth Uhealth & &
\end{tabular}

Legenda: *Dados não encontrados.

Fonte: Silva LVF, et al., 2021. 
Em contraste, dentre os países que publicaram estudos utilizando protocolos de usabilidade, identificaram-se seis $(33,3 \%)$ nos EUA, seguindo-se o Brasil com quatro $(22,2 \%)$, Canadá com dois $(11,1 \%)$, Holanda, Coreia do Sul, Espanha, Alemanha e Turquia, Austrália e a Suécia, cada um com um estudo (5,5\% cada).

A análise dos estudos permitiu apontar as características dos achados em conformidade com o número de participantes, autoria dos artigos, área de aplicação, escalas usadas para verificar a usabilidade e quantificação de estudos validados (Figura 3).

Figura 3 - Mapa Conceitual dos estudos validados, número de participantes, área de aplicação, escalas utilizadas e autoria dos artigos.

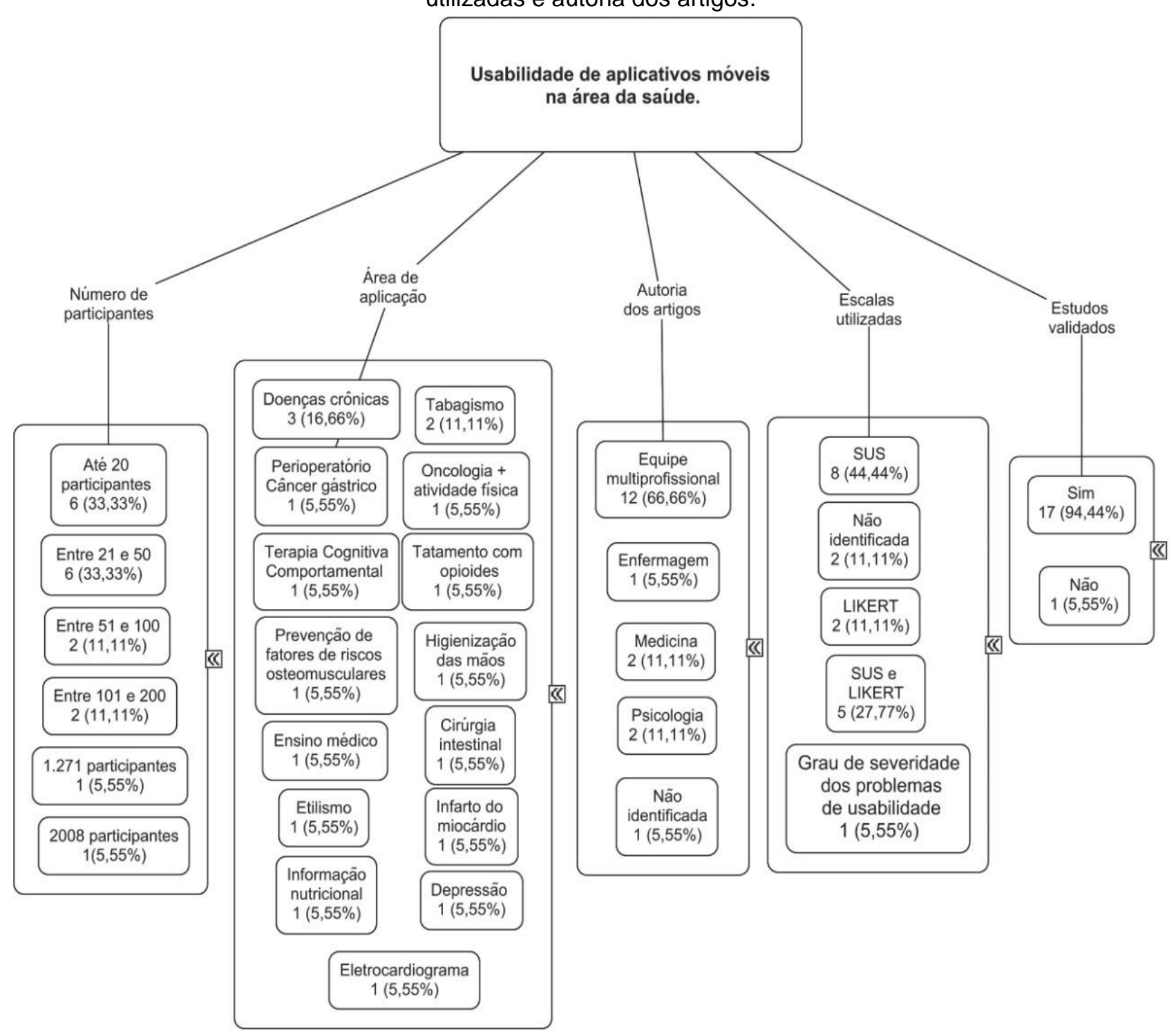

Fonte: Silva LVF, et al., 2021.

Observou-se nos resultados, que um número variável de pesquisadores, utilizaram escalas para testar a usabilidade de App móveis, tendo a maioria dos estudos uma amostra de 20 a 50 usuários. Identificou-se um maior interesse de avaliação dos App para a área das doenças crônicas, e a realização destes estudos, em grande parte, por uma equipe multiprofissional, desvelando a importância do cuidado integral e multifacetado que pode ser ofertado por um App na realização do autocuidado nos domicílios. A escala System Usability Scale (SUS) foi a mais utilizada pelos estudos, e constatou-se que a maioria dos estudos apresentaram-se validos em relação a usabilidade com a aplicação de diferentes formas de análise. 


\section{DISCUSSÃO}

Embora o avanço na ciência venha possibilitando a criação de variados aplicativos voltados para a saúde, verificar a qualidade dessas tecnologias é algo imprescindível para atestar sua distribuição nas diferentes plataformas digitais. Como alternativa, a usabilidade pode ser medida através de instrumentos validados, compreendendo as facilidades na utilização, maneira de interação e obtenção de finalidades específicas ao uso de um App (SANTOS AO, et al., 2019; LIMA CJM, et al., 2019; LAMPRINOS I, et al., 2016).

Protocolos são necessários para avaliação da usabilidade de App, de maneira a rever problemas que possam surgir no uso, o que possibilita melhor versão final para posterior distribuição ao público-alvo, como pacientes ou profissionais de saúde (DE LA VEGA R, et al., 2018). Foi identificada uma maior concentração destes estudos oriundos de fontes internacionais, com idioma inglês e distribuídos na base de dados MEDLINE, configurando maior concentração em publicações sobre a temática abordada. Depreende-se, em conformidade com os descritores escolhidos, maior hegemonia de publicações que tivessem como referencial teórico a usabilidade de aplicativos móveis, nos EUA. O que denota, maior número de artigos científicos publicados em inglês, e a importância do idioma, que se expressa na propagação deste tipo de conhecimento.

O recorte temporal adotado nesta pesquisa, últimos cinco anos, evidenciou ausência de artigos de acordo com os critérios estabelecidos para o ano de 2017, bem como maior volume de publicação no ano de 2019, evidenciando a importância que o tema apresentou nos últimos dois anos. A construção de app em saúde se configura como uma alternativa inovadora de cuidar, e que engloba os diferentes atores em saúde, pois envolve equipes interprofissionais, na busca de um cuidado integral a clientela, muitas vezes não possibilitado em uma consulta de rotina ou durante um período de internação hospitalar (ALVIM AL e COUTO B, 2019)

Neste sentido, disponibilizar aplicativos validados em relação à usabilidade, aspecto avaliado, é imprescindível para assegurar a validade da tecnologia criada, demonstrando a necessidade de rigor na construção de recursos tecnológicos, e que deve ser estimulada como forma inovadora e essencial para quem estuda a temática de construção de App em saúde (DE LA VEGA R, et al., 2018).

No que se refere à disseminação de artigos, os veículos de publicação internacionais foram os responsáveis pela maior parte, com destaque para o jornal acadêmico Thorax, apresentando Qualis/Capes/Brasil - Plataforma Sucupira (2013-2016) A1, considerada a melhor classificação do sistema, bem como maior fator de impacto, 8.834. Trata-se de uma das principais revistas de medicina respiratória do mundo, que publica artigos de pesquisa clínica e experimental em pediatria, medicina respiratória, patologia, imunologia, farmacologia e cirurgia. Ainda foi possível identificar que a maioria dos estudos apresentavam no mínimo o estrato " $A$ " do Qualis/Capes, o que demonstra a importância do rigor metodológico aplicado aos estudos analisados e a importância que a temática vem tomando nos cuidados em saúde.

Dezessete dos 18 estudos foram validados após a avaliação de usabilidade, e uma única pesquisa, ao seu término, concluiu que necessitaria de estudos subsequentes para melhor entendimento dos elementos impactantes do aplicativo, aperfeiçoamento da usabilidade e determinação da eficácia a longo prazo. Tornase importante validar os Apps construídos, especialmente aqueles desenvolvidos para o autocuidado em saúde, uma vez que este tipo de processo permite maior segurança em relação ao conteúdo, viabilidade, aceitabilidade e satisfação da usabilidade do App pelo usuário final, haja vista que o avanço da tecnologia não tem sido proporcional à realização de estudos científicos que se preocupem com estes aspectos, para a maioria dos Apps desenvolvidos e disponibilizados para uso nas plataformas digitais (COX CE, et al., 2019).

Entre as formas de avaliação da usabilidade, o emprego de escalas surge como alternativa, sendo a mais utilizada, a System Usability Scale (SUS), traduzida para o português como Escala de Usabilidade do Sistema. A SUS é originaria do Reino Unido, desenvolvida por John Brooke em 1986, criada com o objetivo de fornecer uma mensuração das percepções subjetivas das pessoas sobre a usabilidade de um sistema. 
Outros pesquisadores com interesse no assunto estudaram a escala ao longo dos anos e a indicam como uma ferramenta válida e eficaz, possibilitando ao usuário avaliar aspectos como eficácia, eficiência e satisfação, sendo a mais utilizada pelos estudos analisados nessa pesquisa, 13 deles (BANGOR A, et al., 2009; BROOKE J, 2013).

Entre os treze, cinco utilizaram em combinação, a escala do tipo Likert. A escala SUS é composta por um questionário com dez itens avaliativos, os itens 1, 3, 5, 7, e 9 (afirmações positivas), neste caso a pontuação é a posição da escala menos 1, e os itens 2, 4, 6, 8 e 10 (afirmações negativas), com pontuação de 5 menos a posição da escala; ao final, soma-se o valor obtido em cada pontuação, multiplica por 2,5 e o resultado é o valor geral da SUS com a pontuação resultante variando de 0 a 100. Logo, os resultados próximos a 100, são positivos (BANGOR A, et al., 2009; BROOKE J, 2013). Em virtude da maioria dos estudos terem sido publicados em periódicos de alto impacto com Qualis/Capes nos melhores estratos, subtende-se que esta escala apresenta rigor metodológico a ser adotado em estudos científicos, que visem avaliar a usabilidade de App em saúde.

Dois estudos usaram a escala Likert (MINEN MT, et al., 2019; AHMED M, et al., 2020). Em contrapartida, outros dois estudos não possuíam escalas de usabilidade definidas, utilizando questionário estruturado para obter respostas dos usuários (DE LA VEGA R, et al., 2018; DANAHER BG, et al., 2019). Um dos estudos avaliou a usabilidade através da escala de Grau de Severidade dos Problemas de Usabilidade (ALVIM AL e COUTO B, 2019).

O estudo possibilitou ainda, identificar a diversidade de escalas de avaliação dos Apps utilizadas nos estudos, associadas a outras ferramentas que facilitam esta avaliação. Como exemplo deste crescente interesse em avaliar os Apps desenvolvidos, mostra-se pesquisa na qual colaboradores buscaram avaliar iterativamente a usabilidade do aplicativo RELAXaHEAD por meio de escala LiKert com a abordagem Think Aloud, na qual, durante entrevistas com especialistas, e ao interagir com o App pensando em voz alta, os comentários dos participantes eram gravados e codificados (MINEN MT, et al., 2019).

O feedback fornecido durante as coletas permitiu modificações e melhorias no RELAXaHEAD (MINEN MT, et al., 2019). A aplicação de escalas de avaliação é um aspecto que deve ser considerado no momento de escolher esta ferramenta para avaliar os Apps desenvolvidos, que deve priorizar escalas validadas psicometricamente e cientificamente, com o intuito tornar confiável aquela avaliação (BANGOR A, et al., 2009; BROOKE J, 2013).

Em conformidade, na caracterização de participantes por estudos, notou-se variações significativas no número de usuários respondentes das pesquisas, com estudos que abordaram 20 pessoas, e em contrapartida, trabalho canadense que entrevistou 2.008 indivíduos. Nesse, um ensaio clínico randomizado, analisou-se a opinião dos consumidores acerca de diferentes versões de um aplicativo sobre entrega de informações nutricionais, e os usuários, ao concluírem as tarefas do aplicativo, eram direcionados para responder a escala de usabilidade (AHMED M, et al., 2020). No que se assemelha, outra pesquisa examinou a eficácia e o padrão de uso de duas intervenções para tabagistas pararem de fumar, compondo sua amostra com 1.271 participantes, que deveriam atender a diversos critérios de elegibilidade (DANAHER BG, et al., 2019).

Ademais, o estudo de caso com participação de sete indivíduos portadores de doença mental grave e tabagistas, com objetivo de avaliar a usabilidade, a experiência e o engajamento dos usuários em relação a um aplicativo que os ensinava a parar de fumar, foi o que teve menor quantitativo (20 participantes) (VILARDAGA R, et al., 2019). Salienta-se, de acordo com os estudos apresentados, que não há uma definição específica de tamanho amostral para considerar um App validado em relação à usabilidade do produto final, porém, observou-se um número de 20 a 50 participantes (usuários finais do App) como suficiente para realização deste tipo de teste.

Quando analisada a área de aplicação dos Apps testados, verificaram-se ampla distribuição e o desenvolvimento de aplicativos nas mais variadas áreas da saúde, sobressaindo três artigos voltados para doenças crônicas. Os quais buscavam melhora na qualidade de vida de jovens com fibromialgia 
(LAMPRINOS I, et al.,2016), o autogerenciamento da diabetes (DE LA VEGA R, et al., 2018), e cuidados voltados a pessoas com enxaqueca (MINEN MT, et al., 2019), seguido de tabagismo com dois (VILARDAGA R, et al., 2019; DANAHER BG, et al., 2019).

Diante do exposto, conhecer e compreender as necessidades de um paciente é fundamental para o desenvolvimento e sucesso de aplicativos na área da saúde, e os profissionais da saúde são as pessoas responsáveis por esse entendimento. Dos 18 estudos, todos foram escritos por autores que apresentavam titulação mínima de mestre, 12 foram realizados por equipes multiprofissionais e apenas um estudo não foi possível identificar a área de atuação dos autores (SOH JY, et al., 2019). Considerando que o autocuidado em saúde é extremamente incentivado pela equipe multiprofissional, e crescentemente estimulado pelo uso de Apps em saúde, o ponto positivo encontrado nessas buscas se reporta à importância de estes Apps serem desenvolvidos, em sua maioria, pela equipe multiprofissional, o que possibilita a riqueza de conteúdo que pode ser ofertada ao usuário destes Apps em relação ao autocuidado em saúde, nas diferentes áreas de conhecimento.

Porém, considerando que a equipe de enfermagem nas unidades de saúde compõe o maior número em recursos humanos, se comparada às demais categorias profissionais, sendo a classe que possui maior contato direto e continuado com os pacientes, o que oportuniza maior destreza para desenvolver estratégias de melhoramento da qualidade de vida, prevenção e promoção da saúde, tornaram-se escassos os achados de estudos desenvolvidos por esses profissionais. É importante incentivá-los a desenvolver estudos de construção e validação de aplicativos, os quais possam ser aplicados em estudos de ensaios clínicos na área da saúde, testando sua validade e eficácia no aprimoramento do autocuidado, tão recomendado por estes profissionais à sua clientela, e que possam ser utilizados como ferramenta de apoio às ações prescritas por esses profissionais como essenciais ao cuidado.

\section{CONSIDERAÇÕES FINAIS}

O processo de avaliação da usabilidade de aplicativos móveis proporciona mais segurança à tecnologia, prova sua eficiência, validade e eficácia, requisitos fundamentais quando se trata de recursos que envolvam a saúde humana. A partir dos dados analisados constatou-se ampla distribuição de publicações que abordam a usabilidade de aplicativos móveis em saúde, com a utilização de diferentes escalas de avaliação, com destaque para a SUS, concluindo-se que essa pode ser uma escala utilizada com segurança pelos pesquisadores que visem validar a usabilidade de App em saúde. Vale salientar, a necessidade de publicações que melhor caracterizem os métodos utilizados para avaliação de usabilidade, bem como publicações voltadas para a Enfermagem.

\section{REFERÊNCIAS}

1. AHMED $M$, et al. A randomized controlled trial examining consumers' perceptions and opinions on using different versions of a FoodFlip@ smartphone application for delivery of nutrition information. Int J Behav Nutr Phys Act, 2020; 17-22.

2. ALVIM AL, COUTO B. Hands Clean - Taxa Automática Para Higienização Das Mãos: Desenvolvimento de Aplicativo Para Controladores de Infecção. Enfermagem em Foco, 2019; 10 (3): 147-151.

3. BANGOR A, et al. Determining what individual SUS scores mean: Adding an adjective ratingscale. J Usabil Stud, 2009; 4(3):114-123.

4. BROOKE J. SUS: A retrospective. Journal of Usability Studies. Vol. 8, p. 29-40, Fevereiro, 2013.

5. COX CE, et al. Effects of mindfulness training programmes delivered by a self-directed mobile app and by telephone compared with an education programme for survivors of critical illness: a pilot randomised clinical trial. Thorax, 2019 74: 33-42.

6. DANAHER BG, et al. Outcomes and Device Usage for Fully Automated Internet Interventions Designed for a Smartphone or Personal Computer: The MobileQuit Smoking Cessation Randomized Controlled Trial. J Med Internet Res, 2019; 21(6): 13290.

7. DE LA VEGA R, et al. Fibroline: A mobile app for improving the quality of life of young people with fibromyalgia. $J$ Health Psychol, 2018; 23(1): 67-78.

8. GAMA LN, TAVARES CMM. Desenvolvimento e avaliação de aplicativo móvel na prevenção de riscos osteomusculares no trabalho de enfermagem. Texto Contexto Enfermagem, 2019; 28 :20180214. 
9. GUEDES VLS. A bibliometria e a gestão da informação e do conhecimento científico e tecnológico: uma revisão da literatura. Ponto de Acesso, 2012; 6(2): 74-109.

10. JOHNSTON N, et al. Effects of interactive patient smartphone support app on drug adherence and lifestyle changes in myocardial infarction patients: A randomized study. Am Heart J, 2016; 178: 85-94.

11. LAMPRINOS I, et al. Modular ICT-based patient empowerment framework for self-management of diabetes: Design perspectives and validation results. Int J Med Inform, 2016; 91:31-43.

12. LIMA CJM, et al. Desenvolvimento e Validação de um Aplicativo Móvel para o Ensino de Eletrocardiograma. Revista brasileira de educação médica, 2019; 43(1): 157-165.

13. MEDEIROS KKAS, et al. Associações entre o Qualis/CAPES e aspectos bibliométricos da produção científica da enfermagem gerontogeriátrica. Rev Rene, 2012; 13(4): 958-68.

14. MINEN MT, et al. User Design and Experience Preferences in a Novel Smartphone Application for Migraine Management: A Think Aloud Study of the RELAXaHEAD Application. Pain Med, 2019; 20(2): 369-377.

15. O'DONNELL R, et al. Entrega de estratégias comportamentais de proteção personalizadas sobre o consumo de álcool por meio de uma intervenção em smartphone: um estudo piloto. Int J Behav Med, 2019; 26: 401-414.

16. ORMEL HL, et al. Self-monitoring physical activity with a smartphone application in cancer patients: a randomized feasibility study (SMART-trial). Support Care Cancer, 2018; 26(11): 3915-3923.

17. PECORELLI N, et al. An app for patient education and self-audit within an enhanced recovery program for bowel surgery: a pilot study assessing validity and usability. Surg Endosc, 2018; 32(5): 2263-2273.

18. SANTOS AO, et al. Desenvolvimento e Avaliação de uma Plataforma Colaborativa Digital para Educação e Tomada de Decisão Médica Baseada em Evidências. Revista brasileira de educação médica, 2019; 43(1): 513-524.

19. SCHUMAN-OLIVIER Z, et al. MySafeRx: a mobile technology platform integrating motivational coaching, adherence monitoring, and electronic pill dispensing for enhancing buprenorphine/naloxone adherence during opioid use disorder treatment: a pilot study. Addict Sci Clin Pract, 2018; 13(1): 21.

20. SILK JS, et al. Using a Smartphone App and Clinician Portal to Enhance Brief Cognitive Behavioral Therapy for Childhood Anxiety Disorders. Behav Ther, 2020; 51(1): 69-84.

21. SOH JY, et al. A Mobile Phone-Based Self-Monitoring Tool for Perioperative Gastric Cancer Patients With Incentive Spirometer: Randomized Controlled Trial. JMIR Mhealth Uhealth, 2019; 7(2): 12204.

22. VILARDAGA R, et al. Formative, multimethod case studies of learn to quit, an acceptance and commitment therapy smoking cessation app designed for people with serious mental illness. Transl Behav Med, 2019; 9(6): 1076-1086. 